\title{
Effect of a blackberry-derived polyphenol enriched beverage on blood pressure: A randomized controlled crossover trial
}

\author{
C. Heneghan, M. Kiely, E. Manning and A.J. Lucey \\ Cork Centre for Vitamin D and Nutrition Research, School of Food \& Nutritional Sciences, University College Cork, \\ Cork, Republic of Ireland
}

This abstract was awarded the student prize for best oral original communication.

Berry fruits are a rich source of nutrients and polyphenols, and there is evidence to support that increased consumption of berries may contribute to the prevention of cardiovascular disease (CVD) through effects on blood pressure (BP), blood lipid profiles and vascular endothelial function ${ }^{(1)}$. To date, the cardio-protective effects of consuming blackberries (Rubus Fruticosus), a fruit native to Ireland, remains to be established.

This 18-wk randomised controlled trial (RCT), with a crossover design, aimed to investigate the effect of consuming a blackberry polyphenol enriched beverage on BP in adults with an elevated systolic BP. Eighty-three healthy, non-smoking adults (46 male; 37 female), aged 45-75 yr with an untreated systolic BP $(130-\leqslant 150 \mathrm{mmHg})$ were recruited. After a 2-wk washout, participants were randomised to consume either a blackberry polyphenol enriched beverage [total polyphenol content: $\sim 700 \mathrm{mg} / \mathrm{gallic}$ acid equivalents (GAE)/250 ml serving/d] or a control, low-dose polyhenol beverage [ $<100 \mathrm{mg}$ GAE/250 ml serving/d] for a 6-wk study period. Following a 2-wk washout, participants switched to the alternate beverage in the second 6-wk study period. Beverages were matched in terms of nutrient profiles and had similar appearance and taste. Participants attended five fasting study visits for the collection of blood samples and the measurement of office BP and central BP (by Vicorder Tonometric device) in accordance with European Society of Hypertension guidelines ${ }^{(2)}$. Data on anthropometrics, dietary control and health and lifestyle information were also collected.

Data from 80 participants (46 male, 34 female) aged $57.7 \pm 6 \mathrm{yr}$ were included in this analysis. At baseline, no significant differences were observed for BP between the treatment and control groups. Office and central systolic BP and diastolic BP did not significantly change over time, all $P>0.05$ (data not shown).

\begin{tabular}{|c|c|c|c|c|c|c|c|c|c|c|}
\hline & \multicolumn{4}{|c|}{ Treatment beverage $(n 80)$} & \multicolumn{4}{|c|}{ Control beverage $(n$ 80) } & \multirow[b]{3}{*}{$P^{a}$} & \multirow[b]{3}{*}{$P^{b}$} \\
\hline & \multicolumn{2}{|c|}{ Baseline } & \multicolumn{2}{|c|}{ Endpoint } & \multicolumn{2}{|c|}{ Baseline } & \multicolumn{2}{|c|}{ Endpoint } & & \\
\hline & $\overline{\text { Mean }}$ & $\overline{\mathrm{SD}}$ & Mean & $\overline{\mathrm{SD}}$ & Mean & $\overline{\mathrm{SD}}$ & Mean & $\mathrm{SD}$ & & \\
\hline Office $B P$ & & & & & & & & & & \\
\hline SBP (mmHg) & 136.8 & $12 \cdot 4$ & 136.9 & $14 \cdot 1$ & $136 \cdot 2$ & $12 \cdot 8$ & 137.0 & $14 \cdot 4$ & 0.836 & 0.835 \\
\hline $\begin{array}{l}\text { DBP }(\mathrm{mmHg}) \\
\text { Central BP }\end{array}$ & 88.9 & 8.7 & 88.9 & $9 \cdot 1$ & 88.6 & $9 \cdot 1$ & $89 \cdot 1$ & $9 \cdot 6$ & 0.457 & 0.448 \\
\hline SBP (mmHg) & $140 \cdot 1$ & $14 \cdot 0$ & $140 \cdot 1$ & 14.8 & 138.6 & 12.7 & $140 \cdot 0$ & 14.3 & 0.583 & 0.590 \\
\hline $\mathrm{DBP}(\mathrm{mmHg})$ & 76.8 & $8 \cdot 1$ & $77 \cdot 2$ & 6.5 & 76.7 & 7.8 & $77 \cdot 3$ & $7 \cdot 2$ & 0.453 & 0.441 \\
\hline
\end{tabular}

Blood pressure (BP), Systolic blood pressure (SBP), diastolic blood pressure (DBP), ${ }^{\mathrm{a}} \mathrm{P}$ value for inter-group comparisons (Independent $\mathrm{t}$ test), ${ }^{\mathrm{b}} \mathrm{P}$ value for ANCOVA adjusting for baseline BP, gender, age and waist circumference.

The mean change in office or central BP during the intervention was not significantly different between the blackberry polyphenol enriched beverage and the control beverage in both unadjusted and adjusted models which accounted for baseline values, gender, age and waist circumference (see Table).

This is the first study to investigate the effect of a blackberry-derived polyphenol enriched beverage on CVD risk. Supplementation with blackberry polyphenols at $\sim 700 \mathrm{mg}$ GAE/d for 6-weeks does not appear to lower BP in adults at risk of developing hypertension.

Trial Registration: clinicaltrials.gov Identifier NCT 02355444.

The Cardio-Rubus project is funded by the Irish Department of Agriculture, Food \& the Marine through the Food Institutional Research Measures (FIRM) programme.

1. Huang H., et al., Effects of Berries Consumption on Cardiovascular Risk Factors: A Meta-analysis with Trial Sequential Analysis of Randomized Controlled Trials. Scientific Reports, 2016. 6: p. 23625.

2. O'Brien E., et al. (2005) on behalf of the European Society of Hypertension Working Group on Blood Pressure Monitoring. Journal of Hypertension, 23(4), 697-701. 